\title{
Visualizing Impact with Publication Data
}

Introduction

The Research Medical Library (RML) contributes to clinical and academic research at MD Anderson Cancer Center by providing resources, education, and services to the faculty and staff. Librarians and scientific editors are experts in navigating the scientific writing and publishing processes. Librarians provide expert searching skills to complete literature and systematic review searches which increase the quality and rigor of the research. ${ }^{1}$ Scientific editors perform substantive and copy editing of journal articles, reports, and original research. Editorial services may improve the acceptance
rate of publications.

The library tracks published materials that are co-authored or contributed to by library staff. These materials are visualized annually on a library dashboard to demonstrate the impact expert library services on publishing at MD

Methods

RML staff created Google Scholar alerts for each editor and librarian who participates in the academic publishing process, in order to capture library staff acknowledgements and coauthorships. Each alert includes name variations, including middle initials and shortened names.

Alerts are received by email and reviewed by administrative staff for accuracy. Applicable Aulcalions are added to an EndNote database. At the end of the fiscal year, the data is downloaded from EndNote to Excel.

The library's Clinical Librarian cleans the data and adds additional data for context. This includes Impact Factors, which are not included in PubMed or Web of Science records, and funding information, which is often inaccurate or Public (Varsion 2021.1.

Library Staff Co-authored and Contributed to 225 Publications in 2020

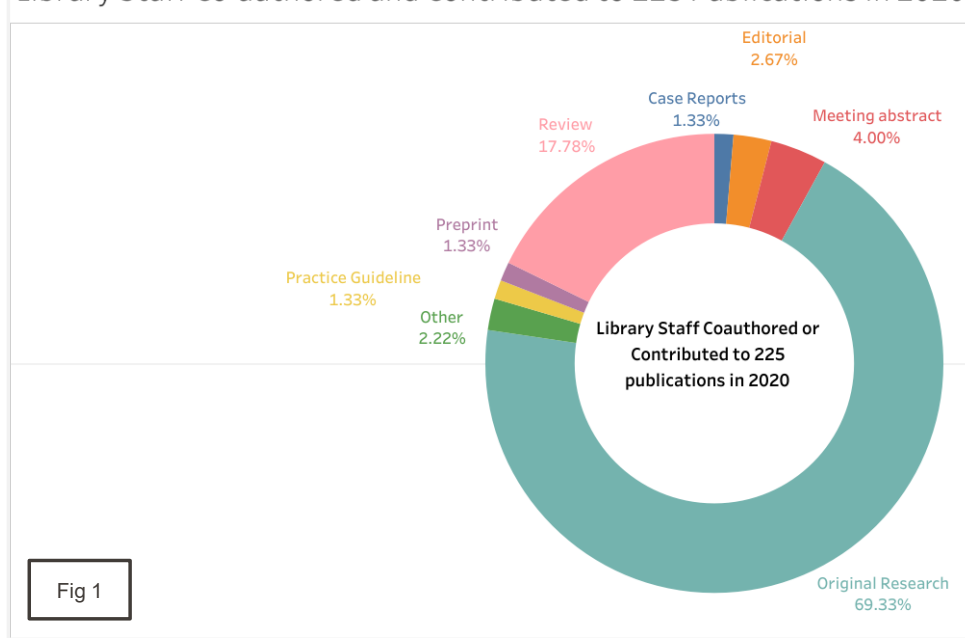

Results

The RML was acknowledged or co-authored on 225 publcations which were published in 142 original research $(69 \%)$, review articles $(18 \%)$, meeting abstracts $(4 \%)$, editorials, case reports, preprints, practice guidelines, and other publications lice a book chapter, a technica note, a book review, and a database review.

$27.6 \%$ of publications supported by RML editors and/or librarians appear in journals with an impact factor above six (Fig 2).

158 of the publications were identified as being supported by federal, institutional, or private funds. At least $10 \%$ of reported publications do not have funding due to the nature of the output. Approximately $46 \%$ of reported funding sources are from the $\mathrm{NIH}$, including the Cancer Center Support Grant (Fig 3). Further research is required to deternin

\section{Conclusion}

The RML's publication dashboard demonstrates the impact and contributions of MD Anderson library staff. More research needs to be completed to show the overall impact on research quality.

https://mdanderson.libguides.com/librarydashboard The authors would like to thank Valerie Felan for her assistance with this project.

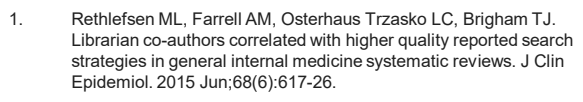

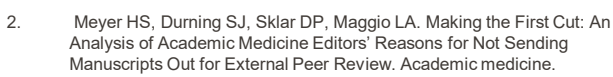

Library Staff Co-authored and Contributed to Publications in 142 Journals in $2020 \cdot 27.6 \%$ of Publications are in Tier 1 or 2 Journals (Impact Factor $>6.0$ )

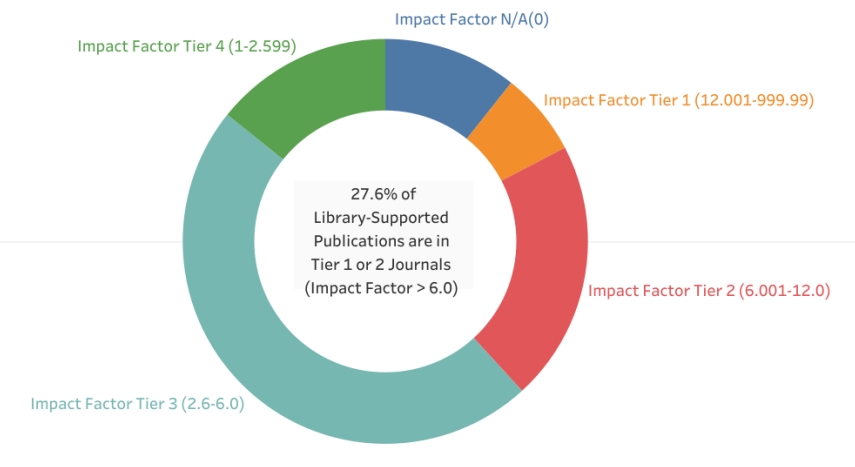

\section{Fig 2}

158 out of 225 RML Coauthored or Contributed Publications in 2020 have a funding source acknowledged. A publication may have multiple funding sources.

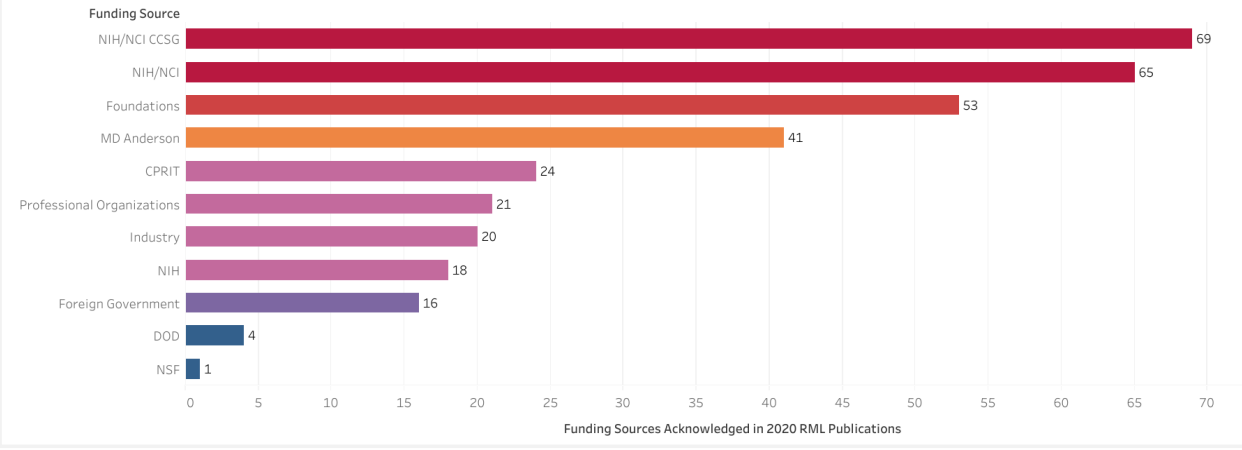

